\title{
Assessment of Antimicrobial Use Pattern Using World Health Organization Prescribing Indicators at a Tertiary Hospital: A Prospective, Observational study
}

\author{
Siavash Shahbazi Nia ${ }^{1 *}$, Shobha Rani R. Hiremath ${ }^{1}$, Shankar Prasad ${ }^{2}$ \\ ${ }^{1}$ Department of Pharmacy Practice, Al-Ameen College of Pharmacy, Bengaluru, India. \\ ${ }^{2}$ Department of General Medicine, St. Philomena's Hospital, Bengaluru, India.
}

\section{ARTICLE INFO \\ Article history: \\ Received on: 08/03/2018 \\ Accepted on: 16/05/2018 \\ Available online: 29/06/2018}

\section{Key words:}

antimicrobial drug

resistance, drug evaluation

studies, Drug Utilizations,

antimicrobial agents.

\begin{abstract}
The use and misuse of antimicrobial agents need to be evaluated since misuse of antimicrobials may lead to increased adverse effects, resistance to antimicrobials, making illnesses more serious, and increasing expenses of health services. The aim of this study was to assess the pattern of antimicrobial prescriptions at a tertiary hospital. This prospective, observational study was conducted at a tertiary hospital after obtaining ethical committee clearance. On a daily basis, data were collected from inpatient case sheets. In addition, some data were obtained from the pharmacy department. According to World Health Organization guidelines, specified indicators were applied and the collected data were analyzed using descriptive statistics parameters. During the study period, 900 patient case sheets were included in the study and it was seen that 255 patients were prescribed antimicrobial agents. It was found that antibiotics were the most common type of antimicrobials prescribed. The average duration of prescribed antimicrobial treatment for each subject was 5.65 days and the average cost of antimicrobials prescribed per patient was 9422 INR (140 USD). It was observed that only for $10.58 \%$ of patients who were prescribed with antimicrobial agents, culture sensitivity test was performed. The use of antimicrobials in our study site was found to be less than optimal. Hence, there is a need to promote rational use of antimicrobials, as their irrational use would lead to antimicrobial resistance.
\end{abstract}

\section{INTRODUCTION}

Universally, an estimated number of 16 million deaths from infections had been reported in 1990. The number of deaths had fallen to 15 million in 2010. The World Health Organization (WHO) anticipates 13 million deaths attributed to such causes in 2050 (Dye, 2014). This decrease in mortality and morbidity rate is due to utilization of antimicrobial drugs, which are one of the most substantial and considerable contributions to therapeutics in the current century (Tripathy, 2008).

Antimicrobial agents account for $20-50 \%$ of total hospital spending on drugs, as they are one of the costliest categories of drug expenditure in hospitals. Various studies report that about $50 \%$ of antimicrobial agents are used inappropriately and they are misused

${ }^{*}$ Corresponding Author

Siavash Shahbazi Nia, Department of Pharmacy Practice, Al-Ameen

College of Pharmacy, Bengaluru, India.

E-mail: siavashshahbazinia6640@gmail.com
(Vlahovic-Palcevski et al., 2000). The misuse and inappropriate use of antimicrobial agents require worldwide attention and immediate action since misuse of antimicrobials may lead to increased adverse effects, resistance to antimicrobials, making illnesses more serious, and increasing expenses of health services (World health organization, 2012; Gizework and Seyfe, 2015). Selection of an antimicrobial agent to treat an infection is far more complicated than matching a drug to a known or suspected pathogen. Ideally, the antimicrobial agent used to treat an infection is selected after the organism is identified and its drug susceptibility established. However, in critically ill patients, such a delay can be fatal and immediate empiric therapy is necessary. The utilization of antimicrobial agents has greatly decreased the morbidity and mortality caused by infectious diseases, but such advances in treatment are being undermined by the rapid growth of antimicrobial resistance, which has become one of the most important issues over the last 20 years (World health organization, 2012; Hosoglu et al., 2013). 
It is very difficult to acquire a trustable estimation of the global burden of antimicrobial resistance since data is not collected systemically and consistently. The burden increases in low-income and middle-income countries like India due to having a less developed healthcare system, higher rates of infectious diseases, low quality and inappropriate use of antimicrobial agents (World trade organization, 2017).

Distinctive demographic profile and geographic position of India presents a unique challenge for management of infectious disease. The country is one of the world's most populated nations, with a considerable proportion of the population living in beggared areas where infectious diseases have the chance to spread exponentially (Chau et al., 2016).

In India, the infectious disease burden is among the highest in the world and mortality rate due to them is 416.75 per 100,000 persons with microbial resistance increasing over time (World trade organization, 2017; Ganesh et al., 2013).

According to World Health Organization, in 2012, infectious diseases were the fourth most commonly occurring diseases in India among which exacerbation of chronic obstructive pulmonary disease and lower respiratory tract infection were the most common causes of death (World health organization, 2017a).

Recent reports presented the inappropriate and irrational use of antimicrobial agents against these diseases, which led to an astounding increment in the rate of development of antimicrobial resistance. In addition, they have shown that inadequacy of public finance has influenced the health sector in India, which in turn results in an accelerated rate of development of antimicrobial resistance (Ganesh et al., 2013).

Recently, World Health Organization (WHO) has reported that there is nominal data on resistant pathogens and antimicrobial resistance in India. So, antimicrobial resistance is of particular concern in India, where spending on healthcare is low and the burden of infectious disease is high. Since the highest microbial disease burden in the world can be observed in India, antimicrobial agents play an important role in restricting morbidity and mortality in the country. The regulation of the medical sector, particularly in prescribing medicines is an important issue that needs to be addressed in India. Lack of knowledge among medical as well as the general public on the rational use of antimicrobials worsens the issue (Das et al., 2017; World health organization, 2017b).

The World Health Organization (WHO) states the necessity of having reliable data on how medicines are used in order to evaluate the accessibility, quality, and cost-effectiveness of care and to determine the problematic areas to develop targeted intervention strategies. As a result, WHO began using specific indicators to evaluate the use of antimicrobial agents in hospitals so as to detect the most common problems arising from antimicrobial use (World health organization, 2012).

In view of all the above, this study was aimed to investigate the use and prescribing patterns of antimicrobial agents by using WHO antimicrobial use indicators. The findings of this study could be habituated to benchmark policy and practice activities regarding the quality of antimicrobial use. Concretely, this insight will avail policy-makers to implement felicitous interventions designed to amend the judicious utilization of antimicrobials in India and more ecumenically.

\section{METHODS AND MATERIALS}

\section{Study setting}

The study was conducted in a tertiary care hospital in Bangalore, India, which is a 400-bed hospital with 18 specialists, 56 physicians, 15 pharmacists and 155 nurses who attend an average of 800 patients per month. Purposively, six wards ( 3 departments) including the department of general medicine (wards B, C, G, and J), surgery department (ward D) and intensive care unit (ICU) were selected to collect the required data. In addition, some part of our data was collected from the Pharmacy Department of the hospital. Each of the wards had at least one pharmacist.

\section{Study design and outcome variable}

It was a prospective, descriptive and observational study, designed according to the objectives of the study. The outcome indicators are related to four general areas of antimicrobial use; hospital indicators, prescribing indicators, patient-care indicators, and supplemental indicators.

\section{Study inclusion/exclusion criteria}

All patients, from both genders and all age groups, admitted to the hospital during the study period from October 2016 to March 2017, were included in our study. All patients of obstetrics and gynecology department (OBG), as well as the patients who received anti-tuberculosis drugs and anti-retroviral drugs, were excluded from the study.

\section{Sampling and data collection}

The standard forms provided by WHO were used for data collection. The guidelines and methods were followed to ensure the reliability of all collected data (World health organization, 2012). The data was collected during a period of 6 months from October 2016 to March 2017.

\section{Hospital indicators}

There are five hospital indicators for which the data was collected during a period of 6 months. The most recent copies of standard treatment guidelines, hospital formulary list, and data on key antimicrobials were acquired from the pharmacy department.

\section{Prescribing, patient-care and supplemental indicators, and prescribing patterns of antimicrobials}

A total of 900 patient case sheets were used to carry out this study. On a daily basis, the data was collected from the patient case sheets, which were prescribed with antimicrobial agents. Both empirically prescribed antimicrobials, as well as those prescribed after culture sensitivity test, were included. Each prescription was reviewed daily until the time of discharge to identify the newly prescribed, substituted or discontinued antimicrobial agents. The patients were followed after discharge to obtain information about their completion of the antimicrobial course.

In this study, from each patient's case sheet prescribed with antimicrobial agent, data such as age of the patient, diagnosis, whether the patient has pneumonia, number of days of hospitalization, whether antimicrobial sensitivity test was done, name of antimicrobial prescribed, whether the antimicrobial agent was prescribed with brand name or generic name, class of 
antimicrobial agent prescribed, whether the prescribed antimicrobial agent was present in hospital formulary list, dosage form, strength, and frequency of prescribed antimicrobial agent, number of doses of antimicrobials prescribed, number of doses of antimicrobials administered, total days of treatment with each antimicrobial agent were collected and entered in standard data collection forms provided by WHO (World health organization, 2012).

\section{Ethical considerations}

The study was ethically cleared by the institutional review board of the hospital (Ethics committee approval number: Al am/2017/113). For using each patient's information in this study, each patient, caregiver or parent was clearly explained about the study and signed informed consent form was obtained from him or her. Each patient, caregiver or parent was assured that the information provided by him or her would be confidential and used only for the purpose of research.

\section{Data analysis}

All the collected data were tabulated and analyzed using descriptive statistics parameters.

\section{RESULTS}

During the study period of 6 months, a total of 900 patients were included in our study. Among these patients, it was found that 255 patients received antimicrobial agents for which 728 times the antimicrobial therapy was prescribed.

\section{Demographic characteristics}

Out of 900 patients included in our study, $49 \%$ were male and $51 \%$ were female. Demographic characteristics of patients are shown in Table 1, indicating that majority of the patients were in the age range of 31-60 years.

Table 1: Demographic characteristics of patients $(n=900)$.

\begin{tabular}{ccc}
\hline Patients variables & \multicolumn{2}{c}{ Results } \\
\hline \multirow{2}{*}{ Age } & $1-30$ years & $152(16.88 \%)$ \\
& $31-60$ years & $413(45.88 \%)$ \\
& $>60$ years & $335(37.24 \%)$ \\
\multirow{2}{*}{ Gender } & Male & $441(49 \%)$ \\
& Female & $459(51 \%)$ \\
\hline
\end{tabular}

\section{Hospital indicators}

The study observations showed that the hospital did have the standard treatment guidelines (STGS) for 50 different infectious diseases. In addition, It was observed that an approved hospital formulary list exists in the hospital, in which 98 antimicrobial agents are included that are identified by both generic names as well as brand names. Among these 98 agents, which must be available in the hospital pharmacy store, $77(78.57 \%)$ antimicrobials were available while remaining $21(21.43 \%)$ antimicrobials were out of stock. The average number of days that a set of key antimicrobials was out of stock was calculated and found to be 6.78 days per month. The hospital purchases of medicines were reviewed and it was found that $23.08 \%$ of total hospital medicine costs during the study period was spent on purchasing antimicrobial agents. The results of hospital indicators are summarized in Table 2.
Table 2: Hospital indicators

\begin{tabular}{|c|c|c|c|}
\hline $\begin{array}{l}\text { SL. } \\
\text { NO. }\end{array}$ & Indicator & Parameter & Result \\
\hline \multirow{2}{*}{1} & \multirow{2}{*}{ Indicator 1} & $\begin{array}{l}\text { The existence of standard treatment } \\
\text { guidelines for infectious diseases }\end{array}$ & Yes \\
\hline & & $\begin{array}{l}\text { Number of infectious diseases for which } \\
\text { the STGS were available }\end{array}$ & 50 \\
\hline \multirow[b]{2}{*}{2} & \multirow[b]{2}{*}{ Indicator 2} & $\begin{array}{l}\text { The existence of an approved hospital } \\
\text { formulary }\end{array}$ & Yes \\
\hline & & $\begin{array}{l}\text { Total number of antimicrobials included in } \\
\text { the approved hospital formulary list or } \\
\text { essential medicine list }\end{array}$ & 98 \\
\hline 3 & Indicator 3 & $\begin{array}{l}\text { Availability of a set of key antimicrobials in } \\
\text { the hospital stores }\end{array}$ & $78.57 \%$ \\
\hline 4 & Indicator 4 & $\begin{array}{l}\text { Average number of days that a set of key } \\
\text { antimicrobials is out of stock }\end{array}$ & $\begin{array}{l}6.78 \text { days } \\
\text { per month }\end{array}$ \\
\hline 5 & Indicator 5 & $\begin{array}{l}\text { Expenditure on antimicrobials as a } \\
\text { percentage of total hospital medicine costs }\end{array}$ & $23.08 \%$ \\
\hline
\end{tabular}

\section{Prescribing indicators}

Out of 900 patients' case sheets, which were reviewed and included in our study, it was found that 255 (28.33\%) patients were prescribed one or more than one antimicrobial agent and for them, the average number of antimicrobials prescribed was 2.85 per patient. The average duration of prescribed antimicrobial treatment for each subject was found to be 5.65 days and the average cost of antimicrobials prescribed per patient was 9422 INR (140 USD). It was observed that all the 728 times the antimicrobial therapy was prescribed were consistent with the hospital formulary list. Only $96(13.18 \%)$ antimicrobial agents were prescribed by generic names and the remaining were prescribed with brand names. In this study, 26 patients were diagnosed with pneumonia among which only $5(19.23 \%)$ patients had been prescribed antimicrobials in accordance with STGS. The results of prescribing indicators are summarized in Table 3.

Table 3: Prescribing indicators.

\begin{tabular}{|c|c|c|c|}
\hline $\begin{array}{l}\text { SL. } \\
\text { NO. }\end{array}$ & Indicator & Parameter & Result \\
\hline 1 & Indicator 6 & $\begin{array}{l}\text { Percentage of hospitalizations with one or more } \\
\text { antimicrobials prescribed }\end{array}$ & $28.33 \%$ \\
\hline 2 & Indicator 7 & $\begin{array}{l}\text { Average number of antimicrobials prescribed } \\
\text { per hospitalization in which antimicrobials } \\
\text { were prescribed }\end{array}$ & 2.85 \\
\hline 3 & Indicator 8 & $\begin{array}{l}\text { Percentage of antimicrobials prescribed } \\
\text { consistent with the hospital formulary list }\end{array}$ & $100 \%$ \\
\hline 4 & Indicator 9 & $\begin{array}{l}\text { The average cost of antimicrobials prescribed } \\
\text { per hospitalization in which antimicrobials } \\
\text { were prescribed }\end{array}$ & $\begin{array}{l}9422 \text { INR } \\
(140 \\
\text { USD)* }\end{array}$ \\
\hline 5 & $\begin{array}{c}\text { Indicator } \\
10\end{array}$ & $\begin{array}{l}\text { The average duration of prescribed } \\
\text { antimicrobial treatment }\end{array}$ & 5.65 days \\
\hline 6 & $\begin{array}{l}\text { Indicator } \\
11\end{array}$ & $\begin{array}{l}\text { Percentage of patients with pneumonia who are } \\
\text { prescribed antimicrobials in accordance with } \\
\text { STGS }\end{array}$ & $19.23 \%$ \\
\hline 7 & $\begin{array}{c}\text { Indicator } \\
12\end{array}$ & $\begin{array}{l}\text { Percentage of antimicrobials prescribed by } \\
\text { generic name }\end{array}$ & $13.18 \%$ \\
\hline
\end{tabular}

\section{Patient care and supplemental indicators}

From the data collected, it was found that a total of 8744 doses of antimicrobial agents were prescribed to the patients 
among which $8715(99.6 \%)$ doses were administered to them properly while $29(0.4 \%)$ doses were missed. During the study period, the patients prescribed with antimicrobial agents were followed to determine the duration of their hospital stay. It was found that each patient was admitted to the hospital for an average of 6.98 days. It was also found that among 255 patients prescribed with antimicrobials, for a total of $27(10.58 \%)$ patients, culture sensitivity test was performed while the remaining patients were prescribed antimicrobials without performing culture sensitivity test. The results of patient care and supplemental indicators are summarized in Table 4.

Table 4: Patient care and supplemental indicators.

\begin{tabular}{cclc}
\hline $\begin{array}{c}\text { SL. } \\
\text { No. }\end{array}$ & Indicator & \multicolumn{1}{c}{ Parameter } & Result \\
\hline 1 & Indicator 13 & $\begin{array}{l}\text { Percentage of doses of prescribed antimicrobials } \\
\text { actually administered } \\
\text { The average duration of hospital stay of patients } \\
\text { who received antimicrobials }\end{array}$ & $99.6 \%$ \\
& Indicator 14 & $\begin{array}{l}\text { Percentage of antimicrobial drug sensitivity } \\
\text { tests reported per hospital admission with } \\
\text { curative antimicrobials prescribed }\end{array}$ & $10.58 \%$ \\
\hline
\end{tabular}

\section{Prescribing pattern of antimicrobials}

Out of 255 patients prescribed with antimicrobial agents, $39(15.3 \%)$ patients received one antimicrobial, 77 $(30.2 \%)$ received two antimicrobials, 70 (27.45\%) patients received three antimicrobial agents and 69 (27.05\%) of the patients received four or more antimicrobial agents. In this study, it was found that among the prescribed antimicrobial agents, antibiotics were the most commonly prescribed antimicrobial agents. Table 5 indicates the distribution of antimicrobial agents based on type and frequency of prescribing. In addition, it was found that majority of antimicrobial agents were prescribed in the department of general medicine. Table 6 represents the distribution of antimicrobial agents in different departments/wards. Different types of infectious diseases were diagnosed in patients prescribed with antimicrobial agents. Table 7 represents the most commonly occurring infectious diseases among our study subjects, as well as the number of antimicrobials that had been prescribed empirically. The most commonly occurring infectious diseases were assessed with reference to the hospital STGS for infectious diseases. It was observed that for patients with diseases like lower respiratory tract infection and exacerbation of the chronic obstructive pulmonary disease, antimicrobial agents were prescribed according to STGS. However, in patients with diseases like urinary tract infections, antimicrobial agents were not prescribed according to STGS. Further, it was also found that among patients with infectious diseases like pneumonia, only for a few patients antimicrobial agents were prescribed according to STGS.

\section{DISCUSSION}

The problems arising from inappropriate use of antimicrobial agents exist worldwide, but such problems are more commonly witnessed in the developing countries where the rate of infection is higher but resources are limited (World health organization, 2012; Okeke et al., 2005). In this study, the use of antimicrobial agents was appraised by applying the specified indicators provided by WHO methodology so as to detect the most common problems arising from antimicrobial use at the study site.

Table 5: Distribution of antimicrobial agents based on type and frequency of prescribing.

\begin{tabular}{ccc}
\hline Type of antimicrobial & $\begin{array}{c}\text { Distribution of } \\
\text { antimicrobial agents }\end{array}$ & $\begin{array}{c}\text { Most common class of } \\
\text { antimicrobial agents }\end{array}$ \\
\hline Antibiotics & $685(94.1 \%)$ & $\begin{array}{c}\text { Cephalosporins } \\
\text { Fluoroquinolones }\end{array}$ \\
Antifungals & $26(3.58 \%)$ & Azoles \\
Antivirals & $17(2.32 \%)$ & Anti-influenza \\
\hline
\end{tabular}

Table 6: Distribution of antimicrobial agents in different departments/wards ( $n$ $=728)$.

\begin{tabular}{ccc}
\hline Department/Ward & $\begin{array}{c}\text { Distribution of } \\
\text { antimicrobial agents }\end{array}$ & Percentage (\%) \\
\hline $\begin{array}{c}\text { General medicine } \\
\text { (wads B, C, G, and J) }\end{array}$ & 505 & 69.4 \\
Surgery department (ward D) & 119 & 16.3 \\
Intensive care unit (ICU) & 104 & 14.3 \\
\hline
\end{tabular}

Table 7: Most common indications for antimicrobial use $(n=255)$.

\begin{tabular}{cc}
\hline Indication & Prevalence \\
\hline Lower respiratory tract infection & $33(12.95 \%)$ \\
Pneumonia & $26(10.2 \%)$ \\
Urinary tract infection & $15(5.9 \%)$ \\
Viral fever & $10(3.92 \%)$ \\
Acute gastroenteritis & $7(2.75 \%)$ \\
Exacerbation of chronic obstructive pulmonary disease & $6(2.35 \%)$ \\
Exacerbation of asthma & $5(1.96 \%)$ \\
Dengue fever & $4(1.57 \%)$ \\
Appendicitis & $4(1.57 \%)$ \\
Pancreatitis & $3(1.17 \%)$ \\
Upper respiratory tract infection & $3(1.17 \%)$ \\
Hepatitis & $2(0.79 \%)$ \\
Empirical therapy & $137(53.7 \%)$ \\
\hline
\end{tabular}

\section{Demographic characteristics}

As a result of biologic differences, social inequities, and restrictive cultural norms, women are at higher risk of developing infectious diseases and have a more severe course of disease compared to men (Gerberding, 2004). In this study, it is observed that similar to the studies conducted in Pakistan and Jamaica, women are more prone to develop an infectious disease and therefore receive more antimicrobial agents than men (Ganesh et al., 2013; Chin et al., 2010). As people age, there will be an increased tendency to develop infectious diseases particularly due to age-related physiological and anatomical changes as well as gradual deterioration of immune system, malnutrition, and epidemiological elements. In this study, patients of all ages were included and it was seen that similar to another study conducted in India, more than half of our study subjects were above 30 years (Sneha et al., 2016).

\section{Hospital indicators}

The existence of formulary list and standard treatment guidelines for infectious diseases in a health care center represents 
the commitment of the health care center to provide high-quality patient care and promote rational use of medicines (World health organization, 2012). In contrast to the study carried out in a tertiary care hospital in Pakistan (Atif et al., 2017), in our study site, there exist standard treatment guidelines for 50 different infectious diseases, which help the general practitioners to fasten the treatment of patients as well as improving their quality of life. The standard treatment guidelines are revised annually. In addition to standard treatment guidelines for infectious diseases, there exists an approved hospital formulary list in which 98 antimicrobial agents are included by both generic and brand names.

In addition to the approved formulary list and standard treatment guidelines for infectious diseases, it is essential that all the key antimicrobial agents should be available at all times in the hospital pharmacy store. The existence of a set of antimicrobials in the hospital store makes it possible to have access to different types of antimicrobial agents for emergency situations. As a result, proper inventory control should be carried out to reduce "out of stock" condition. In our study, it has been observed that from the 98 antimicrobial agents included in the formulary list, the hospital pharmacy store always procures a set of antimicrobial agents accounting for $77(78.57 \%)$ agents. In other words, the remaining 21 agents are always out of stock. Our result value was lesser than the values obtained in another study conducted in Pakistan in which $93.8 \%$ of antimicrobials were available at the hospital pharmacy store (Atif et al., 2017). When some medicines are not available in the hospital pharmacy store and "out of stock" situation occurs, the general practitioners are limited in prescribing appropriate antimicrobial agents in accordance with the formulary list and standard treatment guidelines. In addition, the patients may not be able to acquire their drug of choice or they may have to buy branded medicines. This, in turn, can result in non-adherence to the medication as well as an enhanced economic burden to the patients (World health organization, 2012). According to the information obtained from the hospital pharmacy stores and the hospital formulary list, it was observed that a set of key antimicrobial agents were out of stock for an average of 6.78 days per month which is lesser than the result of another study conducted in Afghanistan stating that a set of key antimicrobial agents were out of stock for an average of 8.7 days per month (Green et al., 2009). High expenditure on antimicrobials as a percentage of total hospital medicine costs can be representative of prescribing of multiple antimicrobials, unjustified use of antimicrobials, or use of expensive, branded antimicrobials (World health organization, 2012). The hospital purchases of medicines were reviewed and it was observed that $23.08 \%$ of total hospital medicine costs during the study period was spent on purchasing antimicrobial agents which is relatively higher than the results reported in the study conducted in Pakistan which was reported to be $12.2 \%$ (Atif et al., 2017).

\section{Prescribing indicators}

During hospitalization, patients may be prescribed more than one antimicrobial agent, which may be appropriate according to the patient condition, but it may also be the result of non-optimal prescribing such as duplication of medicines, irrational use of combination therapy, and frequent and unnecessary alterations in dosage regimens (World health organization, 2012). In this study,
$28.33 \%$ of hospitalized patients were found to receive one or more antimicrobial agents, which is lower than the result of a study conducted in Pakistan (Atif et al., 2017), however, it is more than the value reported in a study conducted India (Selvaraj, 2016). It was also observed that the average number of antimicrobials prescribed per hospitalization was 2.85 , which was slightly higher than the result of the study conducted in Pakistan in which the average number of antimicrobials prescribed was 2.3 (Atif et $a l ., 2016)$. Our results were on par with the results of the study conducted in an Egyptian university hospital in which the average number of antimicrobials prescribed was 2.7 (Osama, 2012). Irrational use of antimicrobials and increased antimicrobial prescribing can lead to antimicrobial resistance. So, in such cases, maximum efforts should be made to minimize prescribing of antimicrobial agents in order to reduce the risk of antimicrobial resistance.

A formulary list is a hospital policy depicting the medicines of choice and is a tool, which helps in optimizing the use of medicines. Lack of awareness of prescribers about the list, unavailability of listed antimicrobials in the list or prescribing antimicrobial agents with brand names while the medicines are stocked and dispensed with generic names may cause nonadherence to such hospital policy. In this study, we have observed that all the 728 times the antimicrobial therapy was prescribed were consistent with the hospital formulary list. It was not comparable with the results of other studies in which all or some of the antimicrobial agents were not prescribed consistent with the hospital formulary list (Atif et al., 2017; Green et al., 2009; Atif et al., 2016).

Inappropriate treatment such as prescribing more number of antimicrobial agents than recommended, prescribing higher doses or longer periods of treatments than required, and prescribing brand name instead of generic antimicrobials may increase the cost of healthcare (World health organization, 2012). During the period of study, it was observed that the average cost of antimicrobials prescribed per hospitalization was 9422 INR (140 USD), which was considerably higher than the value obtained in a study conducted in Pakistan reported to be 5.4 USD (Atif et al., 2017). High health care cost results in high costs passed on patients, which can be an influential factor in promoting poor medication adherence. Medication non-adherence can lead to poor health outcomes, which in turn results in increased service utilization (Aurel and Maura, 2014). The optimal duration of therapy for the majority of the infectious diseases is not yet determined, so presently it is recommended to treat them for at least 7-10 days. A too short course of treatment may promote the emergence of antimicrobial resistant organisms, while, prolonged patient exposure to antimicrobials, enhances the risk of adverse drug reactions, the incidence of antimicrobial resistance, and also unnecessary expenditure on antimicrobials (World health organization, 2012). In this study, the average duration of prescribed antimicrobial treatment was found to be 5.65 days, which is comparable with the result of another study conducted in another tertiary care hospital in which average duration of prescribed antimicrobial treatment was 5.4 days (Atif et al., 2017). By using STGS, most effective treatments are prescribed to the patients and there is a minimized risk of drug-related complications particularly development of adverse drug reactions 
(Gopalakrishnan et al., 2014). It was observed that among the patients prescribed with antimicrobial agents, there were 26 patients diagnosed with pneumonia among which only $19.23 \%$ patients had received antimicrobials in accordance with STGs. In case, the STGs are not available or they are not followed, there is a possibility that the prescribing of antimicrobial agents is less than optimal. This may lead to increased incidences of adverse drug reactions, hospitalizations, and financial burden (World health organization, 2012). According to the US. Food and Drug Administration (FDA), generic medicines tend to be cheaper than their brand-name counterparts while they are similar in dosage form, safety, strength, route of administration, quality, and performance characteristics (U.S. Food and Drug Administration, 2017). In addition, prescribing by generic names instead of brand names often prevents confusion about multiple names for the same product (World health organization, 2012). The percentage of antimicrobial agents prescribed by generic names was found to be $13.18 \%$, which was a much lesser value compared to the results of other studies in which $83.1 \%$ and $100 \%$ of antimicrobial agents were prescribed by generic names (Atif et al., 2016; Osama, 2012).

\section{Patient care and supplemental indicators}

The percentage of doses of prescribed antimicrobial agents, which are actually administered, represents patient medication adherence as well as the level of patient care provided by the nursing staff. Around $99.6 \%$ of the antimicrobial agents prescribed to the patients were administered which is relatively a high value. High level of patient medication adherence and level of patient care provided by the nursing staff guarantees a successful treatment while non-adherence or poor adherence to the medication minimizes the overall effectiveness of healthcare system (WHO, 2017). As a result of inappropriate treatment or incorrect diagnosis, patients may not improve within the recommended period of time. Prolonged patient hospitalization can cause an astounding increment in the cost of therapy and risk of development of nosocomial infections. While, in case of shortterm hospital stay, antimicrobial treatment may be ineffective and therefore there may be a high chance of developing antimicrobial resistance. Our study subjects were admitted to the hospital for an average period of 6.98 days which was relatively a longer duration of hospitalization in comparison with the patients who were the subjects of other studies conducted in Pakistan and India in which the patients were reported to be hospitalized for an average of 6.4 and 5.48 days respectively (Atif et al., 2017; Ambili et al., 2013). An effective antimicrobial agent can be selected if the sensitivity of the infecting organism to possible therapeutic agents is known. The frequency of culture sensitivity tests performed in the hospital indicates its ability to provide rational antimicrobial therapy (World health organization, 2012). Culture sensitivity tests were requested for only $10.5 \%$ of the study patients. This could suggest that the remaining $89.5 \%$ of the patients were prescribed with antimicrobial agents either on the empirical basis. Appropriate empirical antimicrobial therapy may hasten the survival of the patient and may shorten the duration of patient's hospital stay. While, since inappropriate empirical therapy requires subsequent changes in antimicrobial agents prescribed, it can increase the chances of antimicrobial resistance.

\section{Prescribing pattern of antimicrobials}

There is a literature stating that administration rate of antimicrobial agents in developing countries is more than in developed countries (Van Boeckel et al., 2014). India is one of the largest consumers of antimicrobial agents worldwide (Chau et al., 2016). In our study site, out of 255 patients prescribed with antimicrobial agents $15.3 \%$ of the patients received one, $30.2 \%$ received two, $27.45 \%$ received three and $27.05 \%$ of the patients received four or more antimicrobial agents. From the comparison between our study results and the results of three other studies conducted in Pakistan and India it can be understood that in our study site, a higher number of antimicrobial agents had been prescribed to the patients (Atif et al., 2017; Sneha et al., 2016; Atif et al., 2016). A study conducted in China revealed that antibiotics were the most common antimicrobial agents prescribed among which cephalosporin was the most common class of antibiotic prescribed (Wang et al., 2014). This is on par with the result of our study. These results may be due to failure in antibiotic management under the healthcare system, which needs to be further addressed by the hospital. Lower respiratory tract infection and urinary tract infection had been observed to be the most commonly occurring infectious diseases in our study site for which antimicrobial agents were prescribed. In another study carried out in Turkey, the same result has been reported (Ozgenç et al., 2011).

In addition, it was also observed that some diseases like lower respiratory tract infection were prescribed suitable antimicrobial agents according to hospital STGS while some other diseases like urinary tract infection were not prescribed appropriate antimicrobial agents according to hospital STGS. In another study conducted in Turkey, the same results can be observed (Ozgenç et al., 2011). Acceptance of concept of Standard Treatment Guidelines (STGs) for commonly occurring diseases can be helpful in promoting the quality and uniformity of medical care. Popularization and acceptance of STGS can aid medical practitioners in rational prescribing of medicines so as to treat or cure the illnesses with minimum expenses and minimum use of medicines (Gopalakrishnan et al., 2014).

A few constraints, which were noted during this study, were that the study duration was short ( 6 months) and also that it was a single-centered study.

\section{CONCLUSION}

This was a study for appraising the use of antimicrobial agents in a tertiary care hospital, by using WHO methodology. The results of the current study suggest that the prescribing pattern of antimicrobial agents in our study site was less than optimal. After application of specific indicators, we have encountered some issues in prescribing of antimicrobial agents such as the high number of antimicrobials prescribed per patient, the high cost of prescribed antimicrobial agents per patient, prolonged duration of hospitalization, low adherence of physicians to STGS for infectious diseases as well as prescribing the majority of antimicrobials with brand names. It was also seen that majority of antimicrobial prescribing were not based on antimicrobial sensitivity test reports and were prescribed empirically.

Hence, in view of improving the prescribing pattern of antimicrobial agents and to address issues related with antimicrobial prescriptions, a clinical pharmacist can be an ideal 
healthcare professional to assist physicians in prescribing the most appropriate antimicrobial agents, encouraging them to prescribe antimicrobial agents by generic names according to STGS and also to counsel the patients on how to properly administer the medications so as to improve the rational use of antimicrobial agents and also to decrease the risk of antimicrobial resistance. In addition, instead of empirical therapy, adherence of physicians to evidence-based medicine can improve the individual patient's care, which in turn decreases the cost of antimicrobial therapy and minimizes the duration of hospital stay.

\section{ACKNOWLEDGMENTS}

The authors acknowledge Dr. T. Vithya, Dr. Mohammed Kazim Sheriff and faculty of the hospital for their constructive contributions in the conduct of this study.

\section{FINANCIAL SUPPORT AND SPONSORSHIP}

Nil.

\section{CONFLICT OF INTERESTS}

There are no conflicts of interest.

\section{REFERENCES}

Ambili R, Samna S, Gayathri AM, Uma N, Retnavally KG. Antibiotics prescribing pattern in the in-patient departments of a tertiary care hospital. Archives of pharmacy practice, 2013; 4(2):71-76.

Aurel OI, Maura JM. Adherence and health care costs. Risk Manag Healthc Policy, 2014; 7:35-44.

Atif M, Azeem M, Sarwar MR, Shahid S, Javaid S, Ikram H, Bang U, Scahill S. WHO/INRUD prescribing indicators and prescribing trends of antibiotics in the Accident and Emergency Department of Bahawal Victoria Hospital, Pakistan. Springerplus, 2016; 5(1):1928.

Atif M, Azeem M, Saqib A, Scahill S. Investigation of antimicrobial use at a tertiary care hospital in Southern Punjab, Pakistan using WHO methodology. Antimicrob Resist Infect Control, 2017; 6:41.

Chin V, Harding HE, Tennant I, Soogrim D, Gordon-Strachan GM. Dynamics of antibiotic usage in the Intensive Care Unit at the University Hospital of the West Indies. West Indian medical journal, 2010; 59(2):1-10.

Chau B, Padma N, Raina M. 2016. Infectious Disease Outbreaks in India- Challenges and opportunities. School of Public Health and Community Medicine.

Dye C. After 2015: infectious diseases in a new era of health and development. Philos Trans R Soc Lond B Biol Sci, 2014; 369(1645):20130426.

Das B, Chaundhuri S, Srivastava R, Nair GB, Ramamurthy T. Fostering research into antimicrobial resistance in India. BMJ, 2017; 358:j3535.

Gerberding JL. Women and Infectious Diseases. Emerg Infect Dis, 2004; 10(11):1965-1967.

Ganesh KS, Adithan C, Harish BN, Sujatha S, Gautam R, Malini A. Antimicrobial resistance in India: A review. J Nat Sci Biol Med, 2013; 4(2):286-291.

Gopalakrishnan S, Uudayshankar PM, Rama R. Standard Treatment Guidelines in Primary Healthcare Practice. J Family Med Prim Care, 2014; 3(4):424-429.

Gizework A, Seyfe AA. Assessment of the pattern of antibiotics use in pediatrics ward of Dessie Referral Hospital, North East Ethiopia. International journal of medicine and medical sciences, 2015; 7(11):1-7.

Hosoglu S, Parlak Z, Geyik MF, Palanci Y. Critical evaluation of antimicrobial use--A Turkish university hospital example. J Infect Dev Ctries, 2013; 7(11):873-9.
Okeke IN, Laxminarayan R, Bhutta ZA, Duse AG, Jenkins P, O'Brein TF, Pablos-Mendez A, Klugman KP. Antimicrobial resistance in developing countries. Part I: recent trends and current status. Lancet Infect Dis, 2005; 5(8):481-93.

Ozgenç O, Genç VE, Ari AA, Sibel EI, Saçar S, Ozunlu H, Akgul A, Demirturk N, Çetin CB, Sungur M, Coskuner SA, Avci Meltem, Ergonul O. Evaluation of the therapeutic use of antibiotics in Aegean Region hospitals of Turkey: a multicentric study. Indian J Med Microbiol, 2011; 29(2):124-9.

Osama H. Ibrahim M. Evaluation of Drug and Antibiotic Utilization in an Egyptian University Hospital: An Interventional Study. Intern Med, 2012; 2(2):1-3.

Sneha PP, Tejasree B, Krishnakanth PV. Study of prescription patterns of antibiotics in tertiary care hospital. International Journal of Biomedical Research, 2016; 7(6):372-374.

Selvaraj R. Assessment of Antibiotic Prescription Practices in a Tertiary Care Hospital. Journal of Clinical and Biomedical Sciences, 2016; 6(1):20-23.

Tripathy KD. 2008. Essentials of Medical Pharmacology. 6th edition. New Delhi, India: Jaypee Brothers Medical Publishers (P) Ltd; 688-689.

Green T, Omari Z, Siddiqui Z, Anwari J, Noorzaee A. 2009. Afghanistan Medicine Use Study: A Survey of 28 Health Facilities in 5 Provinces. [Online] available at: http://apps.who.int/medicinedocs/ documents/s20272en/s20272en.pdf [accessed 18 November 2017].

U.S. Food and Drug Administration. Drugs, generic drugs: Generic drug facts. 2017. Available at: https://www.fda.gov/Drugs/ ResourcesForYou/Consumers/BuyingUsingMedicineSafely/GenericDrugs/ ucm167991.htm [Accessed 12 November 2017].

Vlahovic-Palcevski V, Morovic M, Palcevski G. Antibiotic utilization at the university hospital after introducing an antibiotic policy. Eur J Clin Pharmacol, 2000; 56(1):97-101.

Van Boeckel TP, Gandra S, Ashok A, Caudron Q, Grenfell BT, Levin SA, Laxminarayan R. Global antibiotic consumption 2000 to 2010: an analysis of national pharmaceutical sales data. Lancet Infect Dis, 2014; 14(8):742-750

World health organization. How to Investigate Antimicrobia Use in Hospitals: Selected Indicators (Second Edition). 2012. Available at: http://apps.who.int/medicinedocs/en/d/Js21031en/ [Accessed 01 October 2016].

Wang YY, Du P, Huang F, Li DJ, Gu J, Shen FM, Jiang YY. Antimicrobial prescribing patterns in a large tertiary hospital in Shanghai, China. International journal of antimicrobial agents, 2014; 48(6):666-673.

WHO. Adherence to long-term therapies: evidence for action. 2017. Available at: http://www.who.int/chp/knowledge/publications/ adherence_report/en/ [Accessed 15 November 2017].

World health organization. India: WHO statistical profile. 2017a. Available at: http://www.who.int/gho/countries/ind.pdf?ua=1 [Accessed 02 March 2017].

World health organization. Combating antimicrobial resistance in India. 2017b. Available at: http://www.searo.who.int/india/topics/ antimicrobial_resistance/Combating_Antimicrobial_Resistance_in_India/ en/ [Accessed 18 November 2017].

World trade organization. Antimicrobial resistance: a global epidemic. 2017. Available at: https://www.wto.org/english/news_e/ news16_e/heal_29aug16_e.pdf [Accessed 15 November 2017].

How to cite this article:

Shahbazi Nia S, Hiremath SRR, Prasad S. Assessment of Antimicrobial Use Pattern Using World Health Organization Prescribing Indicators at a Tertiary Hospital: A Prospective, Observational study. J App Pharm Sci, 2018; 8(06): 132-138. 